\title{
Thinking Process of Student in Mathematic Solving Problem Based on Adversity Quotient
}

\author{
Binur Panjaitan \\ Institut Agama Kristen Negeri Tarutung, Indonesia \\ panjaitan_binur@yahoo.com
}

\begin{abstract}
This article describes thinking process of Junior High school based on three categories of adversity quotient, they are: quitter (low adversity quotient), camper (medium adversity quotient), and climber (high adversity quotien). This research is done by using explorative qualitative for quitter, camper, and climber subject. To collect data used the think alouds method combined with the retrospect method.On the think alouds method the subject is asked to voice, while solving a problem.Subject retrospection was performed after the subject worked on each step given. This research finding find out that there is distinctive thinking process among quitter, camper, and climber subject.
\end{abstract}

Keywords: Problem Solving, Adversity Quotient, Thinking Process.

\section{Introduction}

Each student cannot avoid from mathematic learning difficulty at school. It has to be realized that students experience difficulty in learning mathematic commonly with distinct level of difficulty. Avoiding the difficulty in learning mathematic is one of the pragmatic purposes, getting easy, it meansgetting into foolish, and it will face bigger problem later on. Related with this the students are needed to encourage themselves to fond of mathematic. Students have to think that mathematic is essential. Though this case can be identified students who have high adversity quotient are able to show ability in facing difficulty, in this case in learning mathematic.

Students classified into three categories of Adversity Quotient AQ), they are: quitter (low AQ), camper (medium AQ), and climber (high AQ) [1]. The quitter student is who always avoiding from difficulty. When finding the problem, he chooses decadent and afraid of it. Camper student is somebody who does not want to take biggest risk. He feels satisfy for what have been reached, while the climber student is a person who welcomes the challenge. He can motivate himself then he has high enthusiasmsand always makes everything becomes realized.

Even though AQ is more focused on of how an individual responds difficulty. Succeed people in learning are they who have high AQ [2]. The student whose high AQ has motivation and good achievement [3]. AQ gives a positive influence prospective teacher (university students) mathematical understanding of achievement skills [4].

In learning and solving problem of mathematic, students do thinking process. They think to get the answer. Thinking process is the way of human's mind activity. Entrance information is organized in it, so what have been stored in it need adaptation or changing. This process is known as adaption. Adaptation for new scheme can be done in two ways, they are assimilation and accommodation. It is selected happened based on variety scheme stored into mental. The process of assimilation and accommodation will keephappening until balancing occurred. 
The objective of this research is to describe students' thinking process at the level of quitter, camper, and climber in mathematic solving problem. To avoid the reluctant terms in this research, it is necessary to explain several terms such as:

a) Adversity Quotient (AQ) is the ability to solve the difficulty

b) Thinking process is mental activity happened in the students' mind during solving problem which can be investigated through the process of assimilation, accommodation, and abstraction. Assimilation is directly integration new stimuli into existence scheme. Accommodation is change of old scheme or creating new one in order to fix the structure of received scheme. Abstraction is one of activity which has function to reorganize vertical concept of mathematic constructed previously become one of new mathematic concept which more complex. Reorganize vertical is restructure activity from low level into higher one;

c) Mathematic problem is mathematic item in form of story. Solving mathematic problem are: understanding problem, planning problem solving, doing planning problem solving, recheck problem solving [5].

The significance of this research can give contribution such as: as development of theory of thinking process based on adversity quotient in mathematic problem, as the basic of model of mathematic learning development which concern to Adversity Quotient, as a consideration to plan and do mathematic learning especially mathematic problem solving and consideration of research development related into thinking process based on Adversity Quotient in mathematic solving problem especially.

\section{Methodology}

This research is explorative design with qualitative approach. This research subjects are students of Junior High school at grade VII at Swasta RK BintangTimurPematangsiantar. To group the students into three category AQ such as quitter, camper, and climber, it is needed Questioners of Adversity Response Profile (ARP). After getting ARP scores, continuing to do grouping. The Category as followings: score AQ more than 149 is high AQ, score more than 120 and less than or same with 149 is medium or moderate, while score AQ less than or same with 120 is low [6]. Grouping of students who categories quitter, camper, and climber purposively one for each.To 90 students were given the ARP test so that there were 24 students in the climber group, 36 in the camper group and 40 in the quitter group.

Technique of collecting data is think-alouds and interview. The key instrument in this research is the researcher herself. For that when collecting data in the field, the researcher participated during the research process and followed actively research subjects' activities related into collecting data through interview. Beside of this key instrument, another one is duty of solving problem, mathematic items. Before doing the instrument (items), it is validate previously. It is given for two mathematic teachers and one of mathematic pedagogy expert. Validation is guided into problem, readable, face validity used. 


\section{Result and Discussion}

\subsection{The Thinking Process of Subject S1 (Climber Student)}

$\mathrm{S} 1$ is able in writing fluently and right for what is known and for what asking for the three items written before.S1 is able to integrate perception directly or new experience into scheme in his thought, without using changing, but develops his schemata. Through this condition, can be said that $\mathrm{S} 1$ done assimilation thinking process in understanding problem at item geometry problems (M1), algebra problems (M2), andthe social arithmetic problems (M3). It is relevant into that assimilation is thinking process which integrated by someone through perception, concept, even new experience into scheme or pattern which has been existed in thought, and [7] proposes that assimilation does not cause change of schemata on the other hand develops schemata. In line with this Piaget says that assimilation is the incorporation of new events intelligence as scheme or concept [8].

The arranged planning by S1 at the three items (M1, M2, and M3) has been enough to be guided to complete the items. $\mathrm{S} 1$ is able to receive information from the three items so he can plan problem solving. S1 can integrate perception directly or new experience into scheme has been existed in his thought. It can be said that $\mathrm{S} 1$ does assimilation thinking in planning solving problem at item M1, M2, and M3.

Drawing picture of rectangleto map garden and road in around of it at item M1, indicates that $\mathrm{S} 1$ has done abstraction thinking process by using symbol or picture rectangle to represent garden and road around of it. As Soedjadi proposed that in story item frequently we do abstraction by using symbol ' $x$ ' or ' $y$ 'even another one to represent certain many things.

There are three kinds of abstraction, they are: empirical abstraction, pseudo-empirical abstraction, and reflective abstraction. It is like what Piaget presented that there are three abstractions:empirical abstraction (focusing on objects and their properties), pseudo-empirical abstraction (focusing on action on objects and the properties), and reflective abstraction (focusing on mental objects) [9], [10].

In supposition written previously, symbol of rectangle inside and rectangle outside as garden and the road encircles the garden is mental object, so in this case S1 does reflective abstraction thinking process. It can be concluded that in planning to solve the problem S1 does assimilation thinking process and reflective abstraction.

S1 can do planning to solve problem structurally. S1succeeds to answer the three items rightly, without having signified obstruction. In this case S1 uses assimilation thinking process in problem planning.

Related to planning M2 when S1 implements reflective abstraction thinking process in completing item M2, S1 also has implements reflective abstraction thinking process. The completion has used mental object by using symbol.

It can be said that $\mathrm{S} 1$ or climber student uses assimilation thinking process and reflective abstraction in doing planning to solve the problem.

S1 was able to check the result of three problems fluently. In this case, S1 implemented assimilation thinking process in doing problem planning. Because S1used assimilation thinking process in checking result of problem solving so it can be concluded that climber student implements assimilation thinking process in checking problem solving. S1 checked again the result which achieved by comparing the achieved result using two different ways. Beside of that S1 checked by reviewing the procedure had been done. In this case S1 used reflective abstraction. 


\subsection{Thinking Process of Subject S2 (Camper Students)}

The result of interview showed that S2 was able to mention learnt data and questioned fluently. Then S2 can restated known and questioned in the item. S2 couldbe stated known and questioned data fluently because S2 can identify: known thing by observing question or given problem, and which was questioned by seeing statement and which was questioned by seeing question existed in given problem.

S2 was able to assimilate information that known thing cab be indentified through statement on the given problem, and which is asked can be identified through question and command on the given problem, so S2 was able to understand the problem by stating what has been known and questioned. By this one S1 did assimilation in understanding problem.

From the above analysis, it can be concluded that S2 did reflective abstraction and assimilation in understanding problem. The result of interview showed that S2 could explain planning in solving problem fluently.

S2 arranged planning of problem solving 1 as follows: the first time S2 draw rectangle which is symbolized the surface of fond, then draw bigger rectangle which indicated fond and road around it. Then counting width of the road by decreasing totally width with width of garden was done.

From the analysis above, it can be seen that $\mathrm{S} 2$ did reflective abstraction and assimilation in doing planning of solving problem. The result of written work and interview data showed that $\mathrm{S} 2$ can recheck the result which has been gotten from problem solving.

S2 recheck the received result from solving problem 1 by way as follows. S2 believe that the step which has been done by him to complete the items is right. To make it sure S2 check every line. S2 used two ways, the result is same. So S2 believed that his answer was right (S2 check every line in step of planning completing problem and for completing problem refers tothe planning, during completing process and after getting the final result). S2 believed for the result achieved, because after it has been compared, for two different ways got the same result.

By rechecking the achieved result from completing problem 2, S2 used ways as following. S2 believed that the achieved result has been right, because by using two different ways, firstly by assimilating, then secondly by assimilating, S2 got the same result. Beside of it S2 checked line by line at the step of planning solving problem and at the step of solving problem based on planning during completing process and after getting the final result. Another way to check it, S2 substitute value of variable which achieved into one of equation, and it was fulfill. The S2 substitute equation into another equation and also is fulfill.

To recheck the achieved result from solving problem 3, S2 used ways as following. S2 check line by line at the step problem solving planning and at the step solving problem based on the planning during completing process and after gaining the final result. And after observing line by line the result was right, so S2 believed that the result which has been achieved by him was right.

From the analysis above it can be concluded that S2 did reflective abstractionand assimilation in checking or rechecking the result which has been achieved from problem solving. 


\subsection{Thinking Process of Subject 3 (Quitter Student)}

From the understanding activityproblem S3 for the three problems it can be seen there is integrating scheme directly which had by S3 by achieving new stimuli. In planning completing problem 1, subject S3 thought that will be counted the perimeter of rectangle because in the item there was a sentence which explains that in around of the garden will plant grasses. S3 thought that in around show that which will determine is around of rectangle. In this case the old concept has to be changed and will receive new concept. By this case accommodation was happened that was change of old scheme or create new scheme to fix structure of received scheme.

In planning of solving problem 2, S3 has done assimilation that is old scheme integration with stimuli which came from the item. From the analysis above, it can be concluded that S3 did assimilation, accommodation, and reflective abstraction in solving problem planning.

In completing problem 1 based on the planning, S3 counted perimeter of rectangle and then timed achieved perimeter with the price of grasses which will be planted for every meter quadrate. This way is amiss, started from understanding until planning for solving problem and doing completing is amiss. Subject S3 deceived with word 'around' and thought it become looked perimeter. In this case it has to be happened accommodation that is the existed concept in the subject thought has to be changed and has to receive the right concept.

In solving problem 2, S3 did what had been planned after gaining the result. While S3 said that only one way which is known by him to complete the problem like problem 2 . This point indicated that subject S3 was not motivated to solve the problem by another way and did not have effort to get accurate result with another way. If $\mathrm{S} 3$ complete the item by another way and the result was compared, so it can be one of ways to check achieved result whether already alright or not yet.

For problem 3, S3 completed it based what had been planned before. S3 believed the achieved result had been right because the used procedure had been right. In this case S3 did assimilation, because he had integrated scheme which already existed in his thoughtwith stimuli at the item. From the analysis result of the three items above, so subject S3 or quitter student solving problem based on the planning by using assimilation, accommodation, and reflective abstraction.

In rechecking the result which had been gotten from problem 1, S3 checked in the planning solving problem and the procedures problem solving based on planning after getting completing whether started from understand problem, plan problem solving until do planning, S3 was mistaken. S3 believed that the result had been achieved because it was same with the result which had been checked. S3 did not have another way to recheck achieved result.

Then to recheck the result which had been gained from problem 2, S3 checked step of planning solving problem and the step of solving problem based on planning after having ways of completing. The value of variable recheck and it was already right.

In rechecking the result which had been gained from problem 3 , checked on the step planning solving problem and at the completing problem after having ways of completing. S3 observed line by line from planning of solving problem until to the achieved result. S3 believed the result had been right, because the result permanently same after recounted. 


\section{Conclusion}

\subsection{Thinking Process of Climber Students in Mathematic Solving Problem}

a) Thinking process of climber student in understanding mathematic problem is reflective abstraction at once assimilation. Student climber is able to state what is known and question fluently. Climber student is able to identify what is known by seeing statement on the given problem, and which is asked by seeing question on the given problem.

b) Thinking process of climber student in arranging planning of mathematic solving problem is reflective abstraction at once assimilation. Planning which is arranged by climber students for the three items (M1, M2, M3) has enough to be guided to complete the items. Climber student is able to integrate perception directly or his new experience into scheme already existed in his thought.

c) Thinking process of climber student in doing planning mathematic solving problem is reflective abstraction and assimilation. Climber student is able to do planning solving problem structurally. Climber student succeeds to answer the three items (M1, M2, and M3) rightly without facing useful difficulty. In completing problem M1, climber students plans two different ways. In this case climber student does thinking process assimilation in doing planning solving problem. Related into planning M2 when climber student did thinking reflective abstraction, so when do planning in completing M2, climber student also have done reflective abstraction thinking process.

d) Thinking process of climber student in rechecking achieved result is reflective abstraction and assimilation. Climber student rechecks achieved result by comparing the result by using two different completing ways. Beside of that climber student checks by review the procedure which has been done.

\subsection{Thinking Process of Camper Students in Mathematic Solving Problem}

a) Thinking process camper student in understanding problem is reflective abstraction at once assimilation. Camper student is able to state which known data and asked fluently, because camper student also can indentify: which is known through statement or given problem, and which is asked by seeing the sentence at given problem.

b) Thinking process camper student in arranging planning mathematic solving problem is reflective abstraction at once assimilation, camper student arranges planning of solving problem.

c) Thinking process camper student in arranging planning of mathematic solving problem is reflective abstraction at once assimilation. From the three items (M1, M2, and M3) given, camper student plans the way to complete them by one way only.

d) Thinking process camper student at in rechecking achieved result is reflective abstraction and assimilation. Camper student recheck the achieved result from solving problem by observes line by line. Camper student believes that the step has been done to complete the items is right.

\subsection{Thinking Process of Quitter Students in Mathematic Solving Problem}

a) Thinking process quitter student in understanding mathematic problem is reflective abstraction, assimilation, and accommodation. Quitter student write what is known and asked at the items and often sees item sheet because of forgot. Understanding quitter 
student for what is known does not use question and the sentence longer and what is asked uses question and the sentence shorter.

b) Thinking process quitter student in arranging planning mathematic solving problem is reflective abstraction, assimilation and accommodation. In this case the old concept has to be changed belongs to quitter students and receives new concept. So accommodation is happened to change old scheme or create new scheme to fix the structure achieved scheme.

c) Thinking process quitter student in doing mathematic problem solving is pseudo-empirical abstraction, assimilation, and accommodation. it has to be happened accommodation that is the existed concept already existed in the student thought has to be changed and has to receive the right concept.

d) Thinking process quitter student in rechecking the result which is achieved from mathematic problem solving is pseudo-empirical abstraction, assimilation, and accommodation. In rechecking the result for problem 1, quitter student rechecks at the planning solving problem step and at completing problem based on planning after gaining completing since from understanding problem, planning solving problem, until doing completion of problem, quitter student still wrong. Quitter student believes for the achieved result, because the achieved result same with the result which has been checked. Quitter student does not have effort to recheck the achieved result.

Based on the results of the study recommended as follows:

a) Students need to realize that in themselves there is a potential adversity quotien that can be increased so that it has a high fighting spirit

b) It is better for teachers to always try to increase the adversity quotients of their students and

c) In teaching the teacher should pay attention to students' Adversity Quotient.

\section{References}

[1] Brooks, J.G and Brooks. M.G. In search of understanding: The case for constructivist classroom. Alexandria, VA: The Association for Supervision and Curriculum Development (1993)

[2] Carol S. Dweck, The New Psychology os Success. Stanford: Random House (2006)

[3] D'sousa, R. A studi of Adversity Quotient of Secondary School Students in Relation to Their School Performace and the School Climate. A Dissertation submitted to The University of Mumbai in Partial Fulfillment of the Requiremnts for the Degree of Master of Education. Departement of Education University of Mumbay. (2006)

[4] Gray, E. \& Tall, D. Abstraction as a Natural Process of Mental Compression. Mathematics Education Research Journal. Vol. 19 N0. 2 pp: 23-40 (2007)

[5] Polya, G. How To solve It. (2 ${ }^{\text {nd }}$ ed.). Princeton, $N J$ : Princeton University Press (1973)

[6] Stoltz, P. Adversity Quotient: Turning Obstacles into Opportunities. New York. Wiley (1997). Adversity Quotient: Turning Obstacles into Opportunities. New York. Wiley (2000)

[7] Tall, D. Reflection on APOS theory in Elementary and Advanced Mathematical Thinking. Proceding of the $23^{\text {th }}$ Conference of PME, Hafia Israel, I : 111-118. (1999)

[8] Wadsworth, B.J. Piaget's Theory of Cognitive and Avective Development. New York, Longman (1989)

[9] W Hidayat et al. The influence of adversity quotient on students'mathematical understanding ability. Journal of Physics: Conference Series, Volume 1157, Issue 3(2019) 